\title{
COVID-19 Impact on Pediatric Oncology and Hematology: A Report From the French Society of Pediatric Oncology
}

\author{
Jérémie GAUDICHON ${ }^{1}$, Eric Thebault ${ }^{2}$, Arthur Felix ${ }^{2}$, Aurélie Phulpin ${ }^{3}$, Catherine \\ Paillard $^{4}$, Aurélia Alimi ${ }^{5}$, Benoît Brethon ${ }^{6}$, Elodie Gouache ${ }^{7}$, Sandra Raimbault ${ }^{8}$, Eva De \\ Berranger $^{9}$, Marilyne Poiree ${ }^{10}$, Séverine Bouttefroy ${ }^{11}$, Nicolas André ${ }^{12}$, and Virginie \\ Gandemer ${ }^{13}$ \\ ${ }^{1} \mathrm{CHU}$ de Caen \\ ${ }^{2}$ Gustave Roussy Institute \\ ${ }^{3}$ CHU Nancy \\ ${ }^{4}$ Hopitaux universitaires de Strasbourg \\ ${ }^{5}$ Institut Curie \\ ${ }^{6}$ Hôpital Universitaire Robert-Debré \\ ${ }^{7}$ Hopital Armand-Trousseau \\ ${ }^{8}$ Oscar Lambret Cancer Centre \\ ${ }^{9} \mathrm{CHRU}$ Lille \\ ${ }^{10} \mathrm{CHU}$ Nice, Service d'Hématologie Oncologie Pédiatrique \\ ${ }^{11} \mathrm{IHOPe}$ \\ ${ }^{12}$ Hôpital pour enfants de \\ ${ }^{13} \mathrm{CHU}$ Rennes
}

August 28, 2020

\begin{abstract}
Introduction: Data regarding coronavirus disease 2019 (COVID-19) description are still limited in pediatric oncology. The French society of pediatric oncology (SFCE) has initiated a study to better describe the presentation and evolution of COVID19 in patients followed in French pediatric oncology and hematology wards. Methods: All patients diagnosed with COVID-19 (polymerase chain reaction [PCR] positive for severe acute respiratory syndrome coronavirus 2 [SARS-CoV-2], or positive IgM serology, or chest computed tomography scan and clinical signs typical of COVID-19) and followed in a SFCE center were enrolled. Data from medical records were analyzed for all patients enrolled up to the end of May 2020. Results: Data was available for 37 patients. Thirty-one were children under 18 years of age. Nineteen patients were female. Seventeen patients had a solid tumor, 16 had a hematological malignancy and four recently underwent hematopoietic stem cell transplantation (HSCT) for non-oncological conditions. Twenty-eight patients presented symptoms, most often with fever, cough, rhinorrhea and asthenia. Ground-glass opacities were the most frequent radiological finding with abnormalities mostly bilateral and peripherally distributed. Twenty-four patients received chemotherapy a month prior to COVID-19 diagnosis. Most patients did not require hospitalization. Three patients required oxygen at the time of diagnosis. In total, five patients were admitted in an intensive care unit because of COVID-19 and one died from the disease. Conclusion: Children and young adults infected with SARS-CoV-2 and treated for a cancer and/or with a HSCT may be at risk for severe COVID-19 and should be closely monitored. (NCT04433871)
\end{abstract}

\section{Introduction}


Coronavirus disease 2019 (COVID-19), resulting from severe acute respiratory syndrome coronavirus 2 (SARS-CoV-2) appears to affect children less severely than adults with majority of benign forms and asymptomatic cases[1]. High mortality rate have been recently reported in adult patients with cancer[2,3]. Data is still limited in children with cancer while some reports suggested that they may be quite safe regarding the infection[4-6]. Although recommendations were initially made by the major child cancer organizations, new challenges have emerged[7, 8]. A better description of the disease and its associated clinical and logistical challenges is available. As a result, pediatric oncology departments around the world can develop appropriate strategies $[9]$.

To characterize clinical presentation and outcomes of children, adolescent and young adults with cancer and infected by SARS-CoV-2, the French society of pediatric oncology (Société Française de lutte contre les Cancers et leucémies de l'Enfant et de l'adolescent - SFCE) initiated a study (PEDONCOVID) to retrospectively and prospectively collect data regarding COVID-19 in this specific population. France has been so far one of the most affected countries with currently a total of 188.450 cases and 28.943 deaths[10]. We describe here the detailed data of 37 patients enrolled in French pediatric oncology centers from the SFCE.

\section{Patients and Methods}

All patients followed in a SFCE center and diagnosed with a SARS-CoV-2 infection who had undergone anti-cancer treatment in the past 6 months ago were enrolled in the study. We also included all patients from SFCE centers who underwent a hematopoietic stem-cell transplantation (HSCT) for any reason and with immunosuppressive therapy either ongoing or interrupted less than 6 months prior. Since SFCE centers may provide care in children and adolescents as well as in young adults, there was no limit of age to enroll patients in the study.

Diagnosis criteria for SARS-CoV-2 infection were as follows: (1) biologically proven infection with positive polymerase chain reaction (PCR) or positive specific positive IgM SARS-CoV-2 serology, (2) clinical and radiological diagnosis with at least two (if any contact with a suspected or confirmed COVID-19 case) or three (if no notion of contact) clinical signs (comprising fever, cough, loss of smell, loss of taste, myalgia, chest pain, dyspnea, respiratory distress syndrome, rhinorrhea, diarrhea, headaches, asthenia and skin rash) and computed-tomography $(\mathrm{CT})$-scan signs (ground glass opacities, crazy-paving, linear condensations with a peripheral and/or bilateral distribution) compatible with SARS-CoV-2 infection. All patients and their families were informed of this study. The study was approved by the ethics committee of the coordinating center (N04/2020/ROU). This study is registered in clinicaltrial.gov (NCT04433871).

\section{Results}

Up to the $28^{\text {th }}$ of May 2020, 41 patients with COVID-19 have been declared in SFCE centers. Most of patients were from Eastern France and the region around Paris, consistent with the distribution of the epidemic in whole population (Figure 1). Thirty-seven patients were included in the study. The main characteristics of patients are shown in Table 1. Of the 37 patients, 31 were children under the age of 18 (mean: 11.2 years [1-25]). Nineteen patients (51\%) were female. Seventeen patients had a solid tumor, 16 had a hematological malignancy and four recently underwent HSCT for non-oncological conditions. Nine patients were treated for a cancer relapse. Contact with an infected person was reported for 19 patients, with a median estimated incubation period of 9.5 days (data available for 10 patients). Twenty-eight patients presented symptoms (Table 2). All patients were tested for SARS CoV-2 infection by PCR on a nasopharyngeal swab, which was positive in 34 patients (92\%). Two patients were diagnosed with positive specific IgM serology and one patient was diagnosed upon typical clinical and radiological findings. Thoracic computed tomographyscan was performed in fifteen patients and was abnormal in all but one case. Ground-glass opacities were the most frequent abnormality (9 cases). Abnormalities were mostly bilateral and peripherally distributed. Blood count results were available for 31 patients. Lymphopenia below $0.5 \mathrm{G} / \mathrm{L}$ was found in thirteen patients. Neutropenia below $0.5 \mathrm{G} / \mathrm{L}$ was found in thirteen patients. Six patients presented concomitant neutropenia and lymphopenia. C-reactive protein (CRP) dosage results were available for 24 patients. Fifteen patients had 
an elevated CRP, with a CRP mean level of $41.6 \mathrm{mg} / \mathrm{L}$ [15-280]. Four patients had a CRP above $50 \mathrm{mg} / \mathrm{L}$. Liver enzymes were measured in seventeen patients, nine of whom had elevations greater than twice the upper limit of normal. A co-pathogen was found in blood samples of two patients (one with Staphyloccocus Epidermidis, and one with Epstein-Barr virus). Twenty-four patients received chemotherapy, on average 12.6 days [0-35] prior to COVID-19 diagnosis, with a mean number of drugs of 2.1 [1-5]. Nine patients were currently (or recently) treated with less than $1 \mathrm{mg} / \mathrm{kg} /$ day of corticosteroids, five with cyclosporin A and one with tacrolimus. Most patients did not require hospitalization to manage COVID-19. Seventeen patients received antibiotics, with ten patients presenting febrile neutropenia. Only one patient received remdesivir and three received hydroxychloroquine outside of a clinical trial. Three patients required oxygen at the time of diagnosis and two of them were transferred in an intensive care unit (ICU). In total, five patients were admitted in an ICU because of COVID-19 and one died from the disease. Their main characteristics are described in Table 3. Of note, four patients had been heavily treated for their cancer and/or recently underwent HSCT. Except for these patients, there were only asymptomatic to moderate forms of infection, with a median follow-up of 21 days [0-58]. For the 13 patients for whom PCR had been repeated and this data was available, the mean time to a negative PCR was 16.5 days [7-28]. COVID-19 delayed oncology treatment in 16 patients for an average of 14 days.

\section{Discussion}

The objective of this study was to describe the clinical presentation of COVID-19 in the pediatric oncology and hematology population, not to determine the incidence of COVID-19. Indeed, each center may have different screening strategies and the data collected in this study do not allow for the calculation of the incidence of COVID-19. Our data shows that clinical and radiological descriptions of COVID-19 are quite similar to those reported in adults, and that incubation period or virus clearance appear to be the same in this population as what has been described before. Most of patients developed a mild or even an asymptomatic form of the disease. However, as we previously stated[11] and in contrast with other reports[4-6], we found that patients may be at a higher risk of developing a severe form of COVID-19. Indeed, five patients (15\%) required admission in an intensive care unit (ICU) and one patient died from COVID-19 complications. Among patients with a cancer history, one had high-grade glioma, the others had a relapsed acute lymphoblastic leukemia (ALL) and were highly immunocompromised. The deceased patient was a four-year-old boy undergoing induction chemotherapy for relapsed ALL. His respiratory state worsened and he was admitted in an ICU, where he developed intense macrophage activation syndrome and subsequent complications that led to his death. In the study from Boulad et al., only one patient required a noncritical care hospitalization and the COVID-19 manifestations were mild for most patients[4]. This is concordant with the data from Spain where no severe case was reported[5]. In contrast, in New York, the joint experience form the memorial Sloan Kettering Cancer Center and New York Presbyterian Hospital shows that among 19 patients infected with SARS-CoV-2, five (20\%) required intensive care including mechanical ventilation and a 12-year-old boy with hemoglobinopathy developed acute chest syndrome and died from COVID-19 complications. In Lombardia, Ferrari and al. reported COVID-19 complications in 2/21 patients[12]. We have no clear explanation for such differences between our work and these reports and it may be only due to happenstance. Indeed, ICU admission criteria are likely similar in France and other Western countries and there is no reason that French patients may be more immunocompromised or vulnerable than their US, Spanish or Italian counterparts. In adults, recent reports suggest that patients currently treated for a cancer or with a history of cancer more frequently develop severe COVID-19 compared to general population[2, 13, 14].

Oncologic treatment was delayed in almost half of cases, which indicates that COVID-19 impacted care of patients even if most cases were mild. Of note, some patients had their treatment as planned and did not develop any complication. However, every situation is unique and clinicians who followed these patients made their decision regarding patients' own history and potent risk factors, so that no general recommendation to pursue oncologic treatment may be stated from this study. On the contrary, delaying a non-urgent oncologic treatment appears to be wise in the context of suspected or proven infection, as suggested by most clinicians (in press). Oppositely, delaying the initial management of children with cancer may be dramatic. Thus, in Phildelphia, Ding et al. reported 5 patients who became critically hill because of delay in diagnosis illustrating 
indirect impact on morbidity of COVID-19[7].

A striking difference between our data and the first published reports is the sex ratio[4,5]. Though there were slightly more females than males in our study, our cohort is equilibrated regarding sex distribution. In some reports concerning pediatric oncology populations, there was a strong proportion of males with 5 to 15 times more male than female $[4,5]$. Similarly, Gampel and al. reported that among the 5 patients requiring intensive care, all mere males[15]. Other studies in pediatric general population[16] or adult oncology population[2] did not found this high proportion of males. Thus, we cannot explain the difference between our study and the work from Boulad et al. and de Rojas et al. but there is currently no clue for a specific sex distribution among children and/or oncology patients.

Biological findings are difficult to interpret since a high proportion of patients recently received drugs that could alter blood tests. However, profound neutropenia and profound lymphopenia were found in almost one third of patients. Even if that can be explained by the oncologic treatment received, the infection likely participated and increased the cytopenias. Lymphopenia is common with SARS-CoV-2 infection, and it appears that the deeper the lymphopenia, the more severe the disease[17, 18]. Our cohort is too small to address this question even if two of the five patients admitted in an ICU had profound lymphopenia. For the same reason, it is not possible to draw a conclusion on the association between PCR level and disease severity. However, it should be noted that CRP level was quite low in our cohort and that of the four patients with elevated CRP level above $50 \mathrm{mg} / \mathrm{L}$, two were admitted to an ICU.

Clinicians should pay attention to lung lesions caused by COVID-19 for patients who may receive treatments that could themselves damage lung tissue. Indeed, delaying lung irradiation or administration of drugs with known lung toxicity may be considered for infected patients. However, this delay should not be too long to avoid the risk of progression. In our cohort, the mean delay in treatment administration was 14 days, which appears reasonable regarding both this risk of disease progression and the risk of complications related to COVID-19.

Until we get more data, we advocate not to reassure parents regarding the mild forms of COVID-19 in patients treated for malignancies. We recommend that patients with severe hematological malignancies and/or receiving heavily immunosuppressive treatments should be carefully watched and protected from the COVID-19 risk. This shall be of interest for patients in South Asia and Latin America who are now facing the COVID-19 pandemic.

\section{Conclusion}

We deliver here the first description of the French pediatric oncology and hematology cohort of patients diagnosed with SARS-CoV-2 infection, which to our knowledge is the largest ever published to date. Consistent with previous reports[4,5], relatively few pediatric cancer patients had clinical signs of COVID-19 or tested positive for the virus, Nevertheless, as previously noted[11], some highly immunocompromised patients are at risk of developing severe forms of COVID-19, notably those with hematological malignancies, who have been heavily pretreated and/or with a history of HSCT, which justifies social distancing and specific triage before admission in oncology units.

\section{Conflict of interest statement and funding sources}

None of the authors have a conflict of interest to declare.

This work was supported by a grant from the French ANR (Agence Nationale de la Recherche)

\section{Acknowledgments}

The authors thank Dr Alison Johnson for English proofreading.

The authors are grateful to all collaborators that participate to the elaboration of this cohort: Coralie Mallebranche (CHU Angers), Liana Carausu (CHU Brest), Mathilde Dubrasquet (CHU Tours), Estelle Thebaud (CHU Nantes), Chrystelle Dupraz (CHU Poitiers), Stephane Ducassou (CHU Bordeaux), Marion Gambart 
(CHU Toulouse), Christophe Piguet (CHU Limoges), Stephanie Haouy (CHU Montepellier), Sandrine Thouvenin (CHU Saint-Etienne), Dominique Plantaz (CHU Grenoble), Justyna Kanold (CHU Clermont-Ferrand), Veronique Laithier (CHU Besancon), Claire Briandet (CHU Dijon), Claire Pluchart (CHU Reims), Pascale Schneider (CHU Rouen), Catherine Devoldere (CHU Amiens), Yves Reguerre (CHU La Reunion)

\section{References}

1. Jiatong S, Lanqin L, Wenjun L. COVID-19 epidemic: disease characteristics in children. J Med Virol , 2020 .

2. Assaad S, Avrillon V, Fournier M-L, Mastroianni B, Russias B, Swalduz A, Cassier P, Eberst L, Steineur M-P, Kazes M, Perol M, Michallet A-S, Rey P, Erena-Penet A-S, Morel A, Brahmi M, Dufresne A, Tredan O, Chvetzoff G, Fayette J, Fouchardiere C de la, Ray-Coquard I, Bachelot T, Saintigny P, Tabutin M, Dupre A, Nicolas-Virelizier E, Belhabri A, Roux P-E, Fuhrmann C, Pilleul F, Basle A, Bouhamama A, Galvez C, Herr A-L, Gautier J, Chabaud S, Zrounba P, Perol D, Blay JY. High mortality rate in cancer patients with symptoms of COVID-19 with or without detectable SARS-COV-2 on RT-PCR [Internet]. Eur J Cancer, 2020 0. [cited 2020 Jun 11] Available from: https://www.ejcancer.com/article/S0959-8049(20)30314-2/abstract

3. Zhang L, Zhu F, Xie L, Wang C, Wang J, Chen R, Jia P, Guan HQ, Peng L, Chen Y, Peng P, Zhang P, Chu Q, Shen Q, Wang Y, Xu SY, Zhao JP, Zhou M. Clinical characteristics of COVID-19-infected cancer patients: a retrospective case study in three hospitals within Wuhan, China [Internet]. Ann Oncol, 2020. [cited 2020 Jun 11] Available from: http://www.sciencedirect.com/science/article/pii/S0923753420363833

4. Boulad F, Kamboj M, Bouvier N, Mauguen A, Kung AL. COVID-19 in Children With Cancer in New York City. JAMA Oncol, 2020.

5. de Rojas T, Perez-Martinez A, Cela E, Baragano M, Galan V, Mata C, Pereto A, Madero L. COVID-19 infection in children and adolescents with cancer in Madrid. Pediatr Blood Cancer, 2020 67: e28397.

6. Terenziani M, Massimino M, Biassoni V, Casanova M, Chiaravalli S, Ferrari A, Luksch R, Meazza C, Podda M, Schiavello E, Spreafico F. SARS-CoV-2 disease and children under treatment for cancer. Pediatr Blood Cancer, 2020 e28346.

7. Ding Y-Y, Ramakrishna S, Long AH, Phillips CA, Montiel-Esparza R, Diorio CJ, Bailey LC, Maude SL, Aplenc R, Batra V, Reilly AF, Rheingold SR, Lacayo NJ, Sakamoto KM, Hunger SP. Delayed cancer diagnoses and high mortality in children during the COVID-19 pandemic. Pediatr Blood Cancer, 2020 e28427.

8. Andre N. Covid-19: Breaking bad news with social distancing in pediatric oncology. Pediatr Blood Cancer , 2020 e28524.

9. Sullivan M, Bouffet E, Rodriguez-Galindo C, Luna-Fineman S, Khan MS, Kearns P, Hawkins DS, Challinor J, Morrissey L, Fuchs J, Marcus K, Balduzzi A, Basset-Salom L, Caniza M, Baker JN, Kebudi R, Hessissen L, Sullivan R, Pritchard-Jones K. The COVID-19 pandemic: A rapid global response for children with cancer from SIOP, COG, SIOP-E, SIOP-PODC, IPSO, PROS, CCI, and St Jude Global. Pediatr Blood Cancer, 2020 67: e28409.

10. COVID-19 Map [Internet]. Johns Hopkins Coronavirus Resour Cent [cited 2020 Jun 3] Available from: https://coronavirus.jhu.edu/map.html

11. Andre N, Rouger-Gaudichon J, Brethon B, Phulpin A, Thebault E, Pertuisel S, Gandemer V. COVID-19 in pediatric oncology from French pediatric oncology and hematology centers: High risk of severe forms? Pediatr Blood Cancer, 2020 e28392.

12. Ferrari A, Zecca M, Rizzari C, Porta F, Provenzi M, Marinoni M, Schumacher RF, Luksch R, Terenziani M, Casanova M, Spreafico F, Chiaravalli S, Compagno F, Bruni F, Piccolo C, Bettini L, D'Angio M, Ferrari GM, Biondi A, Massimino M, Balduzzi A. Children with cancer in the time of COVID-19: An 8-week report from the six pediatric onco-hematology centers in Lombardia, Italy. Pediatr Blood Cancer, 2020 67: e28410. 
13. Tang LV, Hu Y. Poor clinical outcomes for patients with cancer during the COVID19 pandemic [Internet]. Lancet Oncol , 2020. [cited 2020 Jun 23] Available from: http://www.sciencedirect.com/science/article/pii/S1470204520303119

14. Liang W, Guan W, Chen R, Wang W, Li J, Xu K, Li C, Ai Q, Lu W, Liang H, Li S, He J. Cancer patients in SARS-CoV-2 infection: a nationwide analysis in China. Lancet Oncol , 2020 21: 335-337.

15. Gampel B, Lucas AGT, Broglie L, Gartrell-Corrado RD, Lee MT, Levine J, Orjuela-Grimm M, Satwani P, Glade-Bender J, Roberts SS. COVID-19 disease in New York City pediatric hematology and oncology patients. Pediatr Blood Cancer n/a: e28420.

16. Dong Y, Mo X, Hu Y, Qi X, Jiang F, Jiang Z, Tong S. Epidemiological Characteristics of 2143 Pediatric Patients With 2019 Coronavirus Disease in China. Pediatrics, 2020.

17. Zhou F, Yu T, Du R, Fan G, Liu Y, Liu Z, Xiang J, Wang Y, Song B, Gu X, Guan L, Wei Y, Li H, Wu $\mathrm{X}, \mathrm{Xu} \mathrm{J}$, Tu S, Zhang Y, Chen H, Cao B. Clinical course and risk factors for mortality of adult inpatients with COVID-19 in Wuhan, China: a retrospective cohort study [Internet]. The Lancet, 2020. [cited 2020 Mar 22] Available from: https://linkinghub.elsevier.com/retrieve/pii/S0140673620305663

18. Suleyman G, Fadel RA, Malette KM, Hammond C, Abdulla H, Entz A, Demertzis Z, Hanna Z, Failla A, Dagher C, Chaudhry Z, Vahia A, Lanfranco OA, Ramesh M, Zervos MJ, Alangaden G, Miller J, Brar I. Clinical Characteristics and Morbidity Associated With Coronavirus Disease 2019 in a Series of Patients in Metropolitan Detroit. JAMA Netw Open , 2020 3: e2012270-e2012270.

\section{Legends}

Table 1: Main characteristics of enrolled patients.

Table 2: Clinical signs of COVID-19 among the 26 symptomatic cases.

Table 3: Clinical characteristics of patients admitted in an intensive care unit for COVID-19

Figure 1: Reported COVID-19 cases in French pediatric oncology and hematology wards up to the 28th of May

\section{Hosted file}

2020_PBC_JR_table_1.docx available at https://authorea.com/users/354596/articles/478110covid-19-impact-on-pediatric-oncology-and-hematology-a-report-from-the-french-societyof-pediatric-oncology

\section{Hosted file}

2020_PBC_JR_table_2.docx available at https://authorea.com/users/354596/articles/478110covid-19-impact-on-pediatric-oncology-and-hematology-a-report-from-the-french-societyof-pediatric-oncology

\section{Hosted file}

2020_PBC_JR_table_3.docx available at https://authorea.com/users/354596/articles/478110covid-19-impact-on-pediatric-oncology-and-hematology-a-report-from-the-french-societyof-pediatric-oncology 
Lille

2

Paris Nancy Strasbourg

Rennes

21

66

Besançon

1

Lyon

1

Nice

Marseille 1 